\title{
Responding to Commercially Sexually Exploited Children (CSEC): A Community Health Center's Journey towards Creating a Primary Care Clinical CSEC Screening Tool in the United States
}

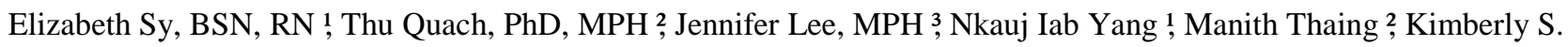 \\ G. Chang, MD, $\mathrm{MPH}^{2}$
}

${ }^{1}$ Banteay Srei, USA.

${ }^{2}$ Asian Health Services, USA.

${ }^{3}$ Association of Asian Pacific Community Health Organizations, USA.

Correspondence: Kimberly S. G. Chang, MD, MPH, Asian Health Services, USA.

Received: April 2, 2016

Accepted: April 25, 2016

Available online: April 26, 2016

doi:10.11114/ijsss.v4i6.1576

URL: http://dx.doi.org/10.11114/ijsss.v4i6.1576

\begin{abstract}
The commercial sexual exploitation of children in the U.S. is an under-recognized yet prevalent issue. An increasing number of Asian-American adolescents, particularly of Southeast Asian descent, are at risk for commercial sexual exploitation due to a myriad of factors. These factors include poverty and intergenerational conflicts as adolescents attempt to adjust to a U.S. lifestyle and culture vastly different from that of their parents. The issues of commercially sexually exploited children (CSEC) first emerged at Asian Health Services (AHS), a federally qualified community health center (FQHC) in Oakland, California, in 2001. AHS Youth Program staff and providers noticed that several Southeast Asian adolescent patients were seeking care for repeated symptoms of sexually transmitted diseases, reporting multiple sexual partners, disclosing chronic truancy issues, revealing a history of sexual abuse, and exhibited other high risk factors for sexual exploitation. Patients eventually disclosed their coercion into the sex trade and shared concerns for their health and safety. In 2006, AHS and its community partner Banteay Srei co-developed the first primary care clinical screening tool in a federally qualified health center. This paper discusses the need for and development process of the screening tool. The process included gathering perspectives of CSEC patients to ensure the tool was grounded in lived experiences of the affected population, and also incorporated insight from healthcare providers and staff to ensure practical implementation by health practitioners. The paper provides recommendations to raise awareness among community members, healthcare professionals, and policy makers in order to curtail the rising CSEC epidemic, and to address the needs of patients who have been commercially sexually exploited.
\end{abstract}

Keywords: commercial sexual exploitation, children, community health center, primary care, screening, domestic minor sex trafficking (DMST), sex trafficking, human trafficking, reproductive health, commercial sexual exploitation of children (CSEC), federally qualified health center (FQHC)

\section{Introduction}

\subsection{The Commercial Sexual Exploitation of Children in the United States}

The commercial sexual exploitation of children in the United States is a prevalent issue that is under recognized. It has been estimated that as many as 300,000 children under the age of 18, are at risk of becoming a commercially sexually exploited victim within the United States (Estes \& Weiner, 2002). However, estimates are often inaccurate due to the reliance on criminal justice statistics, rather than a true measurement of the hidden problem. Furthermore, much of the media coverage of sex trafficking focuses on international cases, effectively eclipsing the prevalence of the issue domestically. In the United States, the underground and transient nature of sexual exploitation has contributed to a lack of understanding amongst service providers and law enforcement. This lack of information has contributed to an often fragmented and uncoordinated response by systems which were not built to recognize commercial sexual exploitation of children as a crime perpetrated against minors, or as child abuse.

The passage of the federal Victims of Trafficking and Violence Protection Act of 2000 finally defined children less than 18 years old engaged in a commercial sexual act as victims of sex trafficking regardless of the use of force, fraud or 
coercion (U.S. Department of State., n.d.). This legislation stipulated protections for this vulnerable population, and has initiated a profound attitudinal shift in the approach to treatment for these youth. Rather than relying on a criminal and juvenile justice frame to arrest and prosecute youth for criminal solicitation, a supportive, health and development-oriented frame is beginning to emerge as a way to provide prevention, intervention and long-term support for these exploited youth (Institute of Medicine; National Research Council, 2013) (Chang et al., 2014).

\subsection{A Community Health Center's Response to Commercial Sexual Exploitation}

The issues of commercially sexually exploited children (CSEC) first emerged at Asian Health Services (AHS) in Oakland, CA, through its youth program and teen clinic. The AHS youth program was established in 1996 to provide reproductive health education in response to increased Asian and Pacific Islander teenage pregnancies in the county. As a result of health education and increased knowledge of reproductive physiology, youth began to request health services, and a confidential teen clinic was started in 1999. In 2001, AHS youth program staff and teen clinic providers noted that some Southeast Asian adolescent patients repeatedly sought reproductive health services for sexually transmitted infection screening and treatment, reported multiple sexual partners, displayed chronic truancy issues, revealed a history of sexual abuse, and exhibited other high risk factors for sexual exploitation (Chang et al., 2014). As staff established rapport and built a therapeutic alliance with their patients, some patients disclosed that they were engaged in the sex trade and talked about their health and safety issues and concerns. In an attempt to access resources for patients, AHS staff connected with other community organizers in the education and youth development fields, and realized that several of these organizers were also actively seeking CSEC resources for their students and clients.

\subsection{Commercial Sexual Exploitation amongst Southeast Asian Youth}

An increasing number of Asian-American adolescents, particularly Southeast Asians (SEA), are at risk for commercial sexual exploitation. SEAs include refugees and immigrants from Cambodia, Laos, Thailand and Vietnam. Many SEA youth are at risk of sexual exploitation due to a myriad of factors including high rates of poverty and cultural and intergenerational conflicts as they attempt to adjust to a U.S. lifestyle vastly different from that of their parents (Chang et al., 2014). A large proportion of first-generation SEA immigrants and refugees suffer the effects of regional conflicts during the Vietnam War. In the Cambodian community, many families still cope with the tragedies of persecution and genocide- post-traumatic stress disorder, depression, addiction, and domestic violence. Studies indicate that $40 \%$ of SEA refugees suffer from depression (Nicholson, 1997). The high rates of poverty among SEA families further exacerbate the problems at home and increase risk of delinquency and risky sexual behavior among SEA teens. With home life struggles, many SEA teens turn to an escape, often re-creating themselves on the streets with their peers (Nicholson, 1997).

In 2004, the rising number of commercially sexually exploited SEA youth in the local area and a lack of culturally informed resources prompted AHS and other community organizers to co-found Banteay Srei, a youth development, asset-building organization that works with young SEA women who are at risk of or engaged in the underground sex trade. The mission of Banteay Srei is to provide resources to support the healthy development of young SEA women at risk of or being sexually exploited, through self-empowerment and self-determination.

\subsection{The Need for Community-based Resources and Screening}

In 2006 to 2011, the Oakland Police Department reported 1,782 sex trafficking cases of minors (H.E.A.T., n.d.). In the time period of 2006-2009, AHS and Banteay Srei participated in the Sexually Abused and Commercially Exploited Youth / Safe Place Alternative (SACEY/SPA) program. The SACEY/SPA program delivered trainings about CSEC recruitment issues and the services offered by AHS and Banteay Srei, and sought referrals from organizations that did not have capacity to serve their CSEC clients. Referrals of CSEC were generated from law enforcement, providers from juvenile justice and child welfare agencies, community workers and health care providers. During Banteay Srei's participation in the SACEY/SPA program, 40 SEA CSEC were referred but only three of these CSEC were referred from law enforcement or had engagement with law enforcement for sexual exploitation (Chang, 2012). This meant $92.5 \%$ of the SEA CSEC assessed by Banteay Srei were referred by community based health providers and support staff, underscoring the limitations of relying on a criminal justice framework to reach these youth. It also highlighted the important role of Banteay Srei's advocacy program in helping CSEC victims navigate systems and access resources. Cultural factors specific to the SEA population place SEA CSEC at a disadvantage for connecting to CSEC resources through local law enforcement. According to the National Center for Prosecution of Child Abuse, families who have experienced historic violence and genocide "often have a fear of law enforcement and are less likely to reach out for help" (Brown, 2011).

Law enforcement and criminal justice agencies have held a critical role in responding to CSEC, but expecting these agencies to respond to the needs of all CSEC is unrealistic and fails to prioritize cultural differences. Institutions and agencies that interact with youth should be able to effectively identify high-risk factors and act from an informed 
response in the event that they encounter a youth who is commercially sexually exploited. Schools, clinics and youth centers that fail to recognize youth that are at risk or are being commercially sexually exploited, miss a critical opportunity to prevent, detect and intervene early in the exploitation process. However, the absence of specific policies and protocols related to commercial sexual exploitation make it difficult for professionals from these sectors to identify and assist CSEC (Institute of Medicine, 2013). A need for guidelines that can direct professionals working with the CSEC population and youth that are at risk for being commercially sexually exploited is crucial to further impact and combat the CSEC epidemic.

\section{Developing a Clinical Screening Tool to Identify CSEC Patients}

\subsection{Gathering Key Information from Stakeholders}

In 2006, Asian Health Services Youth Program and Banteay Srei identified the need for a community-based clinical screening tool that could help to identify CSEC patients. The creation of the screening tool entailed a methodological approach of focused interviews with stakeholders, including the perspectives of CSEC victims as well as health care providers and staff. This ensured that the tool was grounded in the lived experiences of the affected population and that it could be realistically operationalized and implemented by health practitioners. First, one-on-one interviews were conducted with five AHS patients who were identified as CSEC victims to help understand the need for screening at community health center clinical sites. Second, focus groups with health care providers, youth program health educators and outreach staff, and behavioral health staff at AHS were conducted in order to determine risk factors of CSEC patients, and to understand the barriers preventing providers from screening for commercial sexual exploitation within their patient populations. Third, the domestic violence screening and referral protocol, an existing procedure at AHS, was reviewed and provided guidance on how a CSEC screening tool could be developed and implemented throughout the health center.

\subsection{Key Findings from Patients and Healthcare Workers}

The one-on-one interviews with CSEC patients revealed several reasons for the need for clinical screening. There is an increased need for reproductive health services because CSEC are exposed to more sexually transmitted infections (STIs). (Zimmerman, C., Yun, K., Shvab, I., Watts, C., Trappolin, L., Treppete, M.,...Regan, L., 2003), (Institute of Medicine, 2013; National Research Council, 2013), (Crane, P., \& Moreno, M., 2011), (Willis, B., \& Levy, B., 2002). One patient pointed out that clinics were an ideal screening ground because "a healthy body makes money." The patients interviewed mentioned that the adults at AHS' Youth Program and Teen Clinic were some of the more consistent professional adults with whom they interacted. Importantly, one patient noted that compared to all of her interactions with adults in various systems and settings, disclosing her engagement in the sex trade to health providers was a natural extension of answering intimate medical history questions brought up during her health assessment.

Focus groups conducted with providers and community health workers from AHS provided an opportunity to share the high-risk presentations of CSEC patients. AHS Teen Clinic utilizes the HEADSS assessment for every patient at every health visit (Reif \& Elster, 1998). The HEADSS assessment asks patients about their home situation, education status, activities and employment, drug use, sexual activity and risk for suicide. Based on this assessment, providers in the focus group identified several high-risk factors that made them suspect that their patients were being commercially sexually exploited. These high risk indicators included: not living at home but with a "boyfriend", struggling with homelessness issues, chronic truancy, no longer attending school, frequent and consistent requests for STI screenings, disclosing >10 lifetime or casual sexual partners, disclosing history of sexual abuse, and disclosure of friends or family being commercially sexually exploited.

In addition, the focus group identified barriers to screening. Identified barriers included: lack of an integrated protocol, not knowing what to ask, unclear procedures after the patient disclosed commercial sexual exploitation, and fear of causing more harm than good through mandatory reporting. It is worth mentioning that the Institute of Medicine's report Confronting Commercial Sexual Exploitation and Sex Trafficking of Minors in the United States cited mandatory reporting as a barrier to CSEC identification because "a report to law enforcement might lead to a victim's arrest or some form of detention" and "the systems charged with protecting and serving children and adolescents are not designed to support and assist victims and survivors of commercial sexual exploitation effectively" (Institute of Medicine, 2013). 
Table 1. Themes Identified in Focus Groups and One-on-One Interviews

\begin{tabular}{ll}
\hline Stakeholder & Key Information \\
\hline CSEC Patients & $\begin{array}{l}\text { Increased need for sexually transmitted infections (STI) screening due to increased } \\
\text { exposure }\end{array}$
\end{tabular}

More consistent and voluntary contact with healthcare providers compared to other adults from different sectors (i.e. education sector, criminal justice sector)

Disclosure is a natural extension of answering intimate questions regarding sexual health history

AHS Healthcare

Providers and Staff
High-risk indicators include:

Not living at home

Living with a "boyfriend"

Homelessness issues

Chronic truancy

No longer enrolled/attending school

Frequent/consistent requests for STI screenings

$>10$ lifetime sexual partners

History of sexual abuse

Disclosure of friends and family that are CSEC

Other patterns noted by individual providers:

2 or more patients coming in with the same signs and symptoms of a STI, requesting screenings, being positively diagnosed with the same STI

\subsection{Reviewing an Existing Screening Protocol}

The third step of creating the clinical CSEC screening tool consisted of reviewing AHS' domestic violence (DV) screening protocol to identify parallels of how the CSEC screening tool could be effectively developed and utilized. The creation and implementation of the DV protocol highlighted several important steps, including the universal application to perform annually screening of all female patients older than 18 years. The DV protocol clearly outlined steps of what to do when a patient disclosed, and outlined clear supportive referral resources. Additionally, implementation of the DV protocol entailed several trainings to educate staff about the prevalence of DV and the importance of screening. The trainings and awareness building created agency wide support for implementing the DV protocol. Considerations also included input from AHS staff regarding legal requirements surrounding mandatory reporting and suggestions on securing provider buy-in.

\subsection{Drafting a CSEC Screening Tool}

AHS and Banteay Srei drafted a primary care clinical CSEC screening tool and referral protocol in 2007. Before piloting the tool in AHS' Teen Clinic, stakeholders participating in the information gathering sessions were invited to review and suggest changes to the screening tool. One of the suggestions was to craft a more "open" screening question in order to make it easier for patients to seek help or disclose. Based on this suggestion, the screening question was revised to ask patients if they, or someone close to them, were being asked or forced to do something sexual in exchange for money, clothing, a place to stay or something they needed. Notably, the screening tool also included a post-screening protocol providing step-wise guidance on Child Protective Services (CPS) reporting, Oakland Police Department (OPD) reporting, available concrete services and resources with contact information, and what to do if a provider suspected or identified a commercially sexually exploited youth. The screening tool should not be utilized without first noting what to do if there is a positive screen, and what services and resources are in place to ensure a patient's safety and immediate, concrete needs (e.g., shelter, transportation). 


\section{Disseminating the Clinical CSEC Screening Tool}

\subsection{CSEC Screening at AHS}

Once the clinical CSEC screening tool (See Appendix) was created, AHS and Banteay Srei developed and facilitated a series of trainings to over 200 AHS staff members in order to pilot the screening tool. Some of the materials used in the trainings were created during Banteay Srei's involvement in the SACEY/SPA program. Training topics included information on the prevalence of CSEC, how exploiters recruited youth into the sex trade, health and social issues facing commercially sexually exploited children, mandatory reporting requirements, cultural sensitivity and best practices. After implementing the clinical CSEC screening tool in 2008 at AHS, a retrospective study revealed that from 2008-2011, 177 patients had been screened and of those patients 7.3\% disclosed that they were being commercially sexually exploited (Chang, K., Lee, K., Park, T., Sy, E., \& Quach, T., 2015). The retrospective chart review further revealed the clinical indicators of a sexual history which showed significant odds for predicting commercial sexual exploitation (history of STI, sexually active with more than two partners, more than 10 lifetime sexual partners, and more than two combined CSEC risk factors noted in the study) (Chang, 2012).

\subsection{Sharing Resources and Spreading Awareness}

In addition to utilizing the clinical CSEC screening tool at AHS, the tool was disseminated through various trainings convened by AHS and Banteay Srei through the Sexually Exploited Minors Health Committee funded through Measure A in Alameda County, CA. (Measure A, the Essential Health Care Services Initiative, was adopted by Alameda County voters in March 2004. The Measure authorized the County of Alameda to raise its sales tax by one-half cent in order to provide for additional financial support for emergency medical, hospital inpatient, outpatient, public health, mental health and substance abuse services to indigent, low-income, and uninsured adults, children, families, seniors and other residents of Alameda County.) (Alameda County Health Care Services Agency., n.d.) Quarterly SEM Health Committee meetings were held on November 30, 2009, February 22, 2010, March 24, 2010, and May 19, 2010. Local community health centers and agencies including the Native American Health Center, the Oakland Unified School District and Bay Area Legal Aid participated in trainings and received copies of the clinical CSEC screening tool. Representatives from county agencies such as Highland Hospital and the Alameda County Department of Public Health also attended trainings facilitated by AHS and Banteay Srei. The clinical CSEC screening tool was disseminated at other institutions including Kaiser Permanente, the University of California at San Francisco - San Francisco General Hospital, an Office of Women's Health Region IX training coordinated through the California Primary Care Association, and a California Family Health Council training for Title X funded clinics. The CSEC screening tool was made publicly available by internet download through the AHS and Banteay Srei websites, reaching interested parties actively seeking the tool, and has aided in garnering local and national attention to this issue (Chang, et al., 2014; Brown, 2011; Institute of Medicine, 2013). Importantly, this work has raised greater awareness among health care providers, and has been adopted and adapted by other health centers for their own setting (Sexually exploited children protocol, 2013; Mays, 2013).

\section{Conclusion}

\subsection{Serving our CSEC Patients today}

AHS and Banteay Srei continue to bring greater attention and awareness to healthcare providers regarding the commercial sexual exploitation of children. AHS and Banteay Srei conducted a retrospective chart review to estimate the prevalence of CSEC among its patients and to evaluate the impact of the CSEC screening tool in identifying victims (Chang, K., Lee, K., Park, T., Sy, E., \& Quach, T., 2015). As awareness of CSEC issues builds, it is imperative that providers serving this vulnerable population provide input to policy-makers seeking to create effective systems to care for and support these patients. To that end, local, state, and federal efforts should solicit input from direct service providers across sectors to create a comprehensive system of seamless integration of services from prevention to early intervention to long-term support for victims. Endeavors such as the California Child Welfare Council CSEC State Action Team, and the federal Administration of Children \& Families SOAR (Stop. Observe. Ask. Respond to Human Trafficking) to Health and Wellness Network to enhance the health care system's response to human trafficking are current policy efforts which strive to create interdisciplinary solutions and systems to support CSEC.

\subsection{Recommendations}

The commercial sexual exploitation of children is a significant and complex issue in the United States that has reached epidemic proportions (Clawson \& Dutch, 2008). Commercially sexually exploited youth face serious immediate and long-term health problems, which require continuous and integrated coordinated care. In order to more effectively respond to this population, several recommendations should be considered. 
A. A paradigm shift to a public health approach for CSEC care and prevention should be made to guide programs and services, rather than the existing criminal justice framework. This shift is an opportunity to develop programs for prevention, intervention, and long-term care for affected and at risk youth;

B. Implementation of a patient-centered, trauma-informed and culturally competent approach, across all systems serving CSEC;

C. Development of a team-based approach to healthcare services, with funding for enabling services for affected patients (outreach, transportation, interpretation, care coordination, case management). Social determinants contributing to victimization in this population must be addressed for successful prevention, intervention, and long-term recovery for affected patients;

D. Awareness building of CSEC issues across all sectors that serve children. Public awareness campaigns, trainings on CSEC issues and best practices, and the dissemination of these best practices and tools must be made available to the public at large; and

E. Universal screening, creation of tools and protocols for use in systems, institutions and agencies serving children.

Agencies must work together to share resources and coordinate the complex care needs of CSEC patients. All children should be able to access the care and support that they need in order to lead full and healthy lives.

\section{References}

Alameda County Health Care Services Agency. (n.d.) Measure A. Retrieved from https://www.acgov.org/health/indigent/measureA.htm

Brown, P. (2011). In oakland, redefining sex trade workers as abuse victims. New York Times. Retrieved from http://www.nytimes.com/2011/05/24/us/24oakland.html?pagewanted=1\&_r=0

Chang, K. (2012). A community health center model of prevention \& intervention. Presentation delivered at the Institute of Medicine of the National Academies Meeting 3: commercial sexual exploitation and sex trafficking of minors in the united states, San Francisco, CA. Retrieved from http://iom.edu/ /media/Files/Activity Files/Children/SexualExploitationSexTrafficking/Kimberly Changppt Compatibility Mode.pdf

Chang, K., Lee, K., Park, T., Sy, E., \& Quach, T. (2015). Using a clinic-based screening tool for primary care providers to identify commercially sexually exploited children. Journal of Applied Research on Children, 6(1), Article 6.

Chang, K., Sy, E., Vo, T., Nguyen, S., Thaing, M., Lee, J., \& Quach, T. (2014). Framing our response a new approach to care for commercially sexually exploited youth. San Francisco Medicine Journal of the San Francisco Medical Society, 87(1), 21-22.

Clawson, H., \& Dutch, N. (2008). Identifying victims of human trafficking: Inherent challenges and promising strategies from the field., in Issue brief for DHHS. Office of the Assistant Secretary for Planning and Evaluation: Washington, DC.

Crane, P., \& Moreno, M. (2011). Human trafficking: What is the role of the health care provider? Journal of Applied Research on Children, 2(1), 1-27. Retrieved November 25, 2015, from http://digitalcommons.library.tmc.edu/childrenatrisk/vol2/iss $1 / 7$

Estes, R., \& Weiner, N. (2002). The commercial sexual exploitation of children in the U. S., Canada and Mexico. University of Pennsylvania School of Social Work: Philadelphia. H.e.a.t. watch. (n.d.). Retrieved from http://www.heat-watch.org/

Institute of Medicine. (2013). Confronting commercial sexual exploitation and sex trafficking of minors in the united states. Washington, DC: National Academies Press.

Mays, A. (2013). Sexually exploited children screening protocol. Retrieved from http://cshca.wpengine.netdna-cdn.com/wp-content/uploads/2013/03/CSEC-Handout-1.pdf

Nicholson, B. L. (1997). The influence of preemigration and post migrations stressors on mental health: A study of Southeast Asian refugees. Social Work Research, 21(1), 19-31.

Reif, C. J., \& Elster, A. B. (1998). Adolescent preventive services. Primary Care: Clinics in Office Practice, 25(1).

Sexually exploited children screening protocol. (2013). Retrieved from http://cshca.wpengine.netdna-cdn.com/wp-content/uploads/2013/03/CSEC-Handout-1.pdf

U. S. Department of State. (n.d.). Victims of Trafficking and Violence Protection Act of 2000. Retrieved from http://www.state.gov/j/tip/laws/61124.htm 
Willis, B., \& Levy, B. (2002). Child prostitution: Global health burden, research needs, and interventions. The Lancet, $359,1417-22$.

Zimmerman, C., Yun, K., Shvab, I., Watts, C., Trappolin, L., Treppete, M.,...Regan, L. (2003). The health risks and consequences of trafficking in women and adolescents. Findings from a European Study. London: London School of Hygiene and Tropical Medicine.

\section{Appendix}

\section{CSEC SCREENING PROCEDURE GUIDELINE}

Patients ages 11-18 that are exhibiting two or more of the following high risk indicators for sexual exploitation:

- not living at home, living with "boyfriend"

- homelessness issues

- chronic truancy issues

- no longer attending school

- frequent and consistent requests for STI screenings

- sexually active adolescents < 13 years old with $>2$ lifetime or casual sexual partners

- patients who are coming in $>2$ other patients with same s/s of STIs

- $>10$ lifetime or casual partners

- history of sexual abuse

- disclosure of friends or family being sexually exploited

- arriving to appointments with bruises or is intoxicated/smell of drug use

- visual appearance/personality change dramatically

Speak with the person presenting with s/s of sexual exploitation privately and remove others from the room. Use a leading question:

"Over the years, we've noticed that more and more young people are turning to the streets to make money for themselves or for other people. Sometimes patients tell us that:

0 they're exchanging sexual services or 'going on dates' for money, clothes, a place to stay, drugs, etc

0 or in a situation where they're being asked or forced to let other people touch them or do sexual things to them

Many of our patients have told us that they were in a situation like this so we started asking everyone about it, are you, or a close friend of relative, in a situation like this and would like some resources that could help you get out of a situation like this?

We can refer you to an individual that will contact you and help you meet folks who can:

-help you in court

-get reenrolled in school

-help you find a therapist -tell you about programs you might like

-find employment opportunities

-find out if you're eligible for any benefits

(-) If negative note in patient file, screen again in one year.

(?) If questionable assess need follow up during the next visit.

(+) If positive

1. Fill out referral form and contact Banteay Srei Program Manager

2. Let the patient know that someone from Banteay Srei will follow up and make an appointment with patient to talk about resources.

3. Fill Child Protective Services (CPS) form, call and fax in.

4. Debrief during patient checkouts for support.

Created by Asian Health Services and Banteay Srei

\section{(cc) EY}

This work is licensed under a Creative Commons Attribution 3.0 License. 